DOI: $10.34220 / 2311-8873-2021-4-4-64-69$

ТЕПЛООБМЕН В ЗОНЕ КОНТАКТА

ПОВЕРХНОСТЕЙ С ОТКЛОНЕНИЯМИ ФОРМЫ

Попов В.М., Дорняк О.Р., Латынин А.В., Лушникова Е.Н.

Федеральное государственное бюджетное образовательное

учреждение высшего образования «Воронежский государственный

лесотехнический университет имени Г.Ф. Морозова»

E-mail: lat-07@mail.ru

Аннотация: В статье рассматривается тепловая модель процесса теплообмена в составной системе с контактирующими металлическими поверхностями, имеющими макроотклонения формы в виде выпуклостей. Показано, что разработанные ранее методики расчета контактных термосопротивлений пригодны лишь для контактных пар с плоскошероховатыми поверхностями. Для рассматриваемых контактных пар при выводе аналитических зависимостей необходимо учитывать влияние эквивалентной неплоскостности, наличие которой в значительной степени повышает контактное термосопротивление, что требует в процессе проектирования теплонапряженных систем учитывать влияние отклонений формы поверхностей контакта.

Ключевые слова: контактное термосопротивление, макроотклонение формы поверхностей контакта, выпуклость, нагрузка.

\title{
HEAT EXCHANGE IN THE AREA OF SURFACE CONTACT WITH SHAPE DEVIATIONS
}

Popov V. M., Dorniak O. R., Latynin A. V., Lushnikova E. N.

Federal State Budgetary Educational Institution of Higher Education

«Voronezh State Forestry University. G.F. Morozova»

E-mail: lat-07@mail.ru

Summary: The article discusses a thermal model of the heat transfer process in a composite system with contacting metal surfaces with macrodeviations in the form of bulges. It is shown that the previously developed methods for calculating contact thermal resistances are suitable only for contact pairs with flat-rough surfaces. For the considered contact pairs, when deriving analytical dependences, it is necessary to take into account the effect of equivalent non-flatness, the presence of which significantly increases the contact thermal resistance, which requires taking into account the influence of deviations in the shape of the contact surfaces in the process of designing heat-stressed systems.

Keywords: contact thermal resistance, macrodeviations of the contact surfaces shape, convexity, load. 
Создание теплонапряженных систем с составными элементами требуют наличия информации о протекании процессов теплообмена через контактные соединения. Проектировщики вынуждены использовать информацию о формировании термосопротивлений в зоне раздела между отдельными элементами конструкций.

На сегодняшний день накоплен солидный банк экспериментальных и теоретических данных из проведенных исследований отечественных и зарубежных ученых - теплофизиков [1-4].

Однако, несмотря на многочисленные исследования по контактному теплообмену остаются мало- или вообще неисследованными такие вопросы как влияние макроотклонений и особенно в виде неплоскостностей (выпуклостей) контакта соприкасающихся поверхностей [5].

Экспериментальные данные по контактному термосопротивлению (КТС), полученные к настоящему времени для таких соединений носят познавательный характер и свидетельствуют о значительном влиянии на КТС наличия на поверхностях контакта отклонений формы [6-8]. Такого рода соединения особенно часто встречаются в системах с развернутой поверхностью контакта, что характерно для конструкций авиационных и космических летательных аппаратов, при металлообработке, в энергетических объектах и системах военного назначения.

Для такого рода тепломеханических контактов традиционный модельный подход практически неприемлем. Более рациональным и близким к реальным условиям контакта следует признать так называемый эффект двойного стягивания теплового потока к контактной зоне [9-10], когда тепловой поток вначале стягивается к макроконтактам и затем к микроконтактам.

Для реализации тепловой модели двойного стягивания теплового потока примем за основу согласно [2] уравнение

$$
R_{M M}=R_{C T M}+R_{M U},
$$

где $R_{C \text { м м }}$ и $R_{\text {м ш }}-$ термосопротивления стягивания потока к макро и микроконтактам.

Преобразование уравнения тепловой модели двойного стягивания теплового потока для расчета соединений с выпуклостью, имеет следующие ограничения:

- микроконтакты радиуса $a_{\amalg}$ расположены в зоне площадки радиусом $a_{M}$;

- круговой цилиндрический канал теплового потока с радиусом $a_{M}$ коак- 
сиально связан с макроконтактом;

- обязательное условие соблюдения $l$ - длины канала, при котором $l \geq b_{\text {м }}$;

- поверхности рассматриваемого схематичного цилиндра, кроме зоны контакта, адиабатны.

Получим

$$
R_{M M}=R_{C T M}+R_{M W}=\frac{\pi \varphi_{\text {макр }} b_{M}}{2 \bar{\lambda}_{M} \eta_{2}{ }^{1 / 2}}+\frac{\pi a_{M}}{2 \bar{\lambda}_{M} \eta_{3}}
$$

Здесь $\eta_{2}$ и $\eta_{3}$ - относительные площади контактов [11]; $\varphi_{\text {макр. }}-$ коэффициент стягивания теплового потока.

Термосопротивление стягивания теплового потока, после учета озвученных допущений, примет вид [5]

$$
R_{M}=\frac{\pi r_{0}^{2}}{4 \bar{\lambda}_{M} \cdot a} \varphi
$$

Здесь $r_{0}$ - радиус гипотетического контактного элемента;

$\bar{\lambda}_{M}=\frac{2 \lambda_{M 1} \lambda_{M 2}}{\lambda_{M 1}+\lambda_{M 2}}-$ приведенный коэффициент теплопроводности материалов контактной пары;

$\varphi$ - коэффициент стягивания теплового потока.

Условие равномерного распределения площадок макроконтактов по номинальной поверхности касания при воздействии малых нагрузок в реальных соединениях практически недостижимо. Следовательно, необходимо провести преобразования с учетом наличия макроконтактов.

Преобразовав первое слагаемое уравнения (1) согласно [1], получим $\frac{b_{M}}{R_{C T M} \bar{\lambda}_{M}}=\Phi\left(\eta_{2}\right)$ при $\eta_{2}<0,5$ или выразив термосопротивление через эквивалентную толщину, имеем

$$
\frac{b_{M}}{R_{C T M} \bar{\lambda}_{M}}=\frac{\Delta_{M}}{b_{M}}=\frac{1}{\Phi\left(\eta_{2}\right)}
$$

Полагая, что соблюдается равенство $\frac{d i}{b_{M}}=\frac{b_{M}}{2 r_{M i}}(i=1$ или 2$)$, преобразуем уравнения 3 и 4 к виду

$$
\eta_{2}=1,85\left[(P / E)\left(b_{M} / \bar{d}\right)\right]^{2 / 3},
$$


где $P$ - нагрузка; $E$ - модуль Юнга.

Для сферической и плоской поверхностей контакта и для сферических поверхностей.

$$
\eta_{2}=2,88\left[(P / E)\left(b_{M} / \bar{d}\right)\right]^{4 / 5},
$$

Корректность полученных аналитических зависимость апробировалась путем постановки натурных экспериментов с контактными парами из сплава Д16Т и нержавеющей стали марки 1X18Н9Т.

Исследования по определению значений термосопротивлений для контактных пар с плоскошероховатыми поверхностями и имеющими выпуклости проводились на установке, предназначенной для исследования контактного теплообмена в вакууме [1].

Зависимости, полученные в результате эксперимента, показаны на рисунках 1 и 2 в виде зависимостей безразмерного термосопротивления $\Delta_{M} / b_{M}$ от относительной контурной площади $\eta_{2}$ контакта.

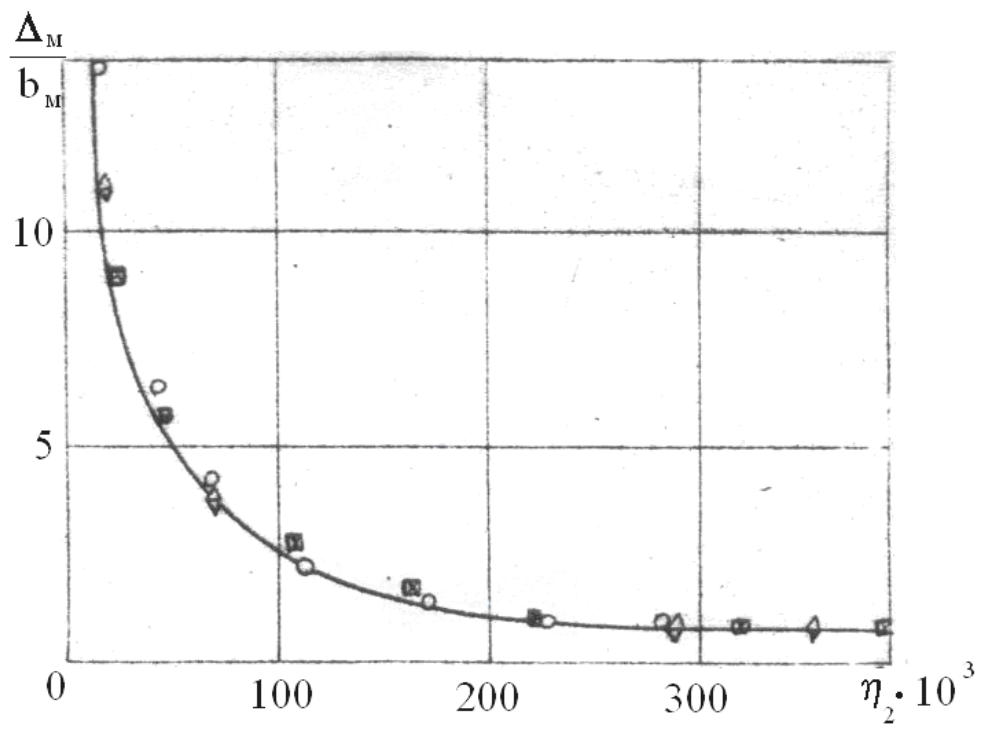

Рисунок 1 - Сравнение теоретических и экспериментальных данных для контактной пары из 1Х18Н9Т с поверхностями, имеющими выпуклость

\begin{tabular}{|c|c|c|c|c|c|}
\hline Обозначения & $\begin{array}{c}\text { Вид обра- } \\
\text { ботки }\end{array}$ & $\bar{d} / b_{M}^{*} \cdot 10^{5}$ & $\left(h_{\max 1} \cdot h_{\max 2}\right) \cdot 10^{6}, \mathbf{M}$ & $\begin{array}{c}T_{K}, \\
\mathrm{~K}\end{array}$ & $\begin{array}{c}\text { Условия } \\
\text { контакта }\end{array}$ \\
\hline$\otimes$ & шлифование & 121 & 3,$2 ; \quad 2,4$ & 373 & нагружение \\
$\circ$ & шлифование & 285 & 3,$8 ; 2$ & 373 & нагружение \\
$\ominus$ & шлифование & 285 & 3,$8 ; 2$ & 558 & нагружение \\
\hline
\end{tabular}

$* b_{M}=15 \cdot 10^{-3} \mathrm{M} ;-$ теоретическая кривая 


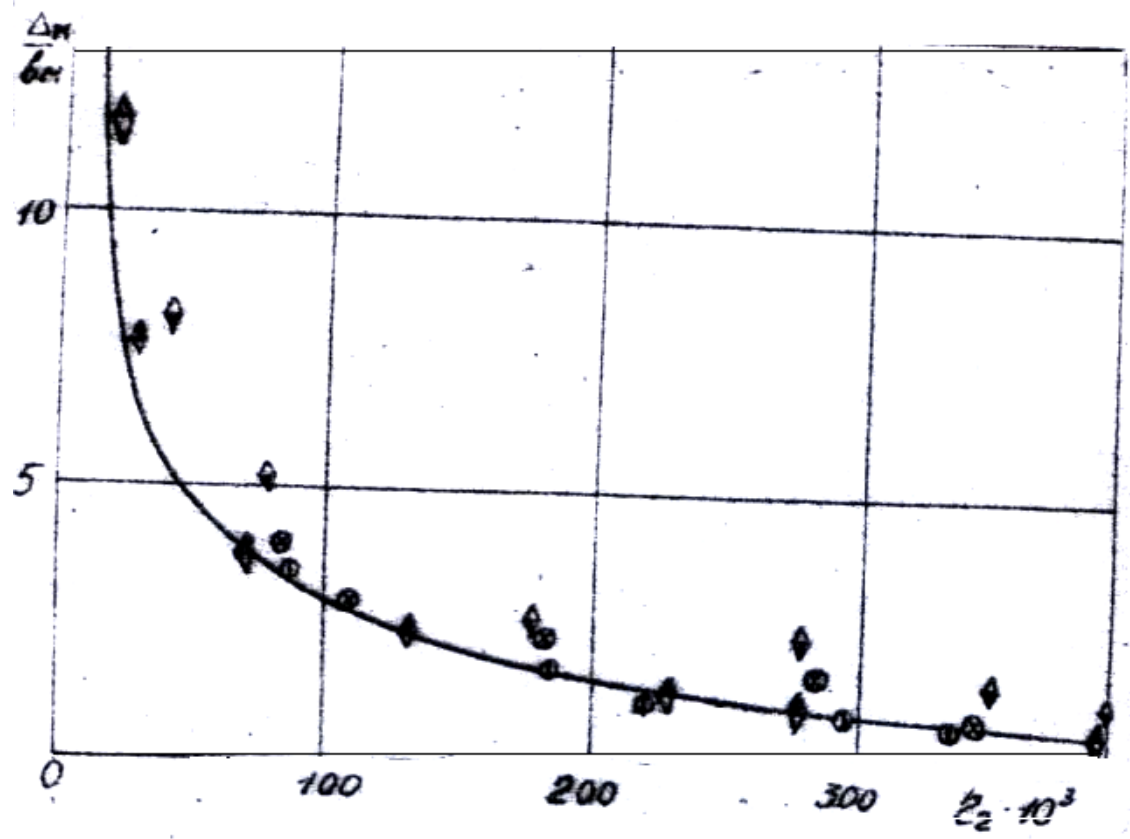

Рисунок 2 - Сравнение теоретических и экспериментальных данных для контактной пары из Д16Т с поверхностями, имеющими выпуклость

\begin{tabular}{|c|c|c|c|c|c|}
\hline Обозначения & Вид обработки & $\bar{d} / b_{M}^{*} \cdot 10^{5}$ & $\left(h_{\max 1} \cdot h_{\max 2}\right) \cdot 10^{6}, \mathrm{M}$ & $\begin{array}{l}T_{K}, \\
\mathrm{~K}\end{array}$ & Условия контакта \\
\hline$\otimes$ & шлифование & 62 & 7,$3 ; \quad 6,8$ & 373 & нагружение \\
\hline$\Phi$ & $\begin{array}{c}\text { Шлифование и } \\
\text { доводка на по- } \\
\text { лировальном } \\
\text { круге }\end{array}$ & 62 & 1,$4 ; \quad 1,2$ & 375 & нагружение \\
\hline$\Delta$ & $\begin{array}{c}\text { Строгание и } \\
\text { доводка }\end{array}$ & 261 & 1,$8 ; 2,3$ & 375 & нагружение \\
\hline$\diamond$ & $\begin{array}{c}\text { Строгание и } \\
\text { доводка }\end{array}$ & 261 & то же & 375 & $\begin{array}{c}\text { на поверхности } \\
\text { заметная окисная } \\
\text { пленка }\end{array}$ \\
\hline
\end{tabular}

$* b_{M}=15 \cdot 10^{-3} \mathrm{M} ;-$ теоретическая кривая

Из сравнения приведенных на рисунках 1 и 2 опытных и теоретических данных видна удовлетворительная сходимость. Следует также отметить, что на изменение величины эквивалентной неплоскости термосопротивление чувствительно реагирует. Отсюда же следует вывод о необходимости при проведение тепловых расчетов в составных системах с поверхностями, имеющими выраженную выпуклость, учитывать специфику формирования контактных термосопротивлений. 


\section{СПИСОК ЛИТЕРАТУРЫ}

1 Шлыков, Ю. П. Контактное термическое сопротивление / Ю. П. Шлыков, Е. А.Ганин, С. Н. Царевский // М. : Энергия, 1977. - 328 с.

2 ПоповВ, В. М. Теплообмен в зоне контакта разъемных и неразъемных соединений /В. М. Попов // М. : Энергия, 1971. - 216 с.

3 Bahrami M., Culham J. R., Javanovich M. M., Schneider G. E. Thermal Contact Resistance of Nonconforming Rough Surfaces, Part 2 : Thermal Model // Journal of Thermophusics and Heat Transfer. Vol. 18, № 2, 2004, - Pp. 218-227. DOI : $10.2514 / 1.2664$.

4 Меснянкин, С. Ю. Современный взгляд на проблемы теплового контактирования твердых тел / С. Ю. Меснянкин, А. Г. Викулов, Д. Г. Викулов // Успехи физических наук., 2009. - Т. 179. № 9. - С.945-970.

5 Weils N. D., Ryder E. A., Thermal resistance of Joints formed be tureen stationary metal Surfaces : Trans of the ASME, 1999. Vol. 71. № 3. - Pp. 259-266.

6 Madhusudana C. V. Thermal Contact Conductance : Sydney: Springer, 2014. $-260 \mathrm{p}$.

7 Somers R. R., Miller J. W., Fletcher L. S. The thermal contact conductance of dissimilar metals // AIAA PAP, № 873,1978, - p. 1-11.

8 Clausing M. Joint Heat Mass Transfer 9. 1966, - 791.

9 Clausing A. M., Chao B. T. Univ of Jllinois End. Exp. Sta. Report, TN-242. August 1963.

10 Клаузин А. М., Чао Б. Т. Термическое сопротивление контакта в вакууме. Теплопередача. Труды американского общества механиков. 1965. № 2.

11 Измайлов, В. В. Контакт твердых тел и его проводимость / В. В. Измайлов, М. В. Новоселова // Тверь : ТГТУ, 2010. - 112 с.

Работа выполнена при финансовой поддержке РФФИ (грант № 20-08-00165). 